\title{
Evaluation for CpG island methylation of FASN and SREBP promoter in Mongolian gerbil NAFLD model
}

Jiusheng $\mathrm{Wu}^{1}$, Yuehuan Liu

${ }^{1}$ College of Animal Sciences, Zhejiang University, Zhejiang, China

${ }^{2} \mathrm{Hangzhou}$ Medical College, Hangzhou, China

Submitted: 9 September 2019; Accepted: 1 April 2020

Online publication: 5 April 2021

Arch Med Sci

DOI: https://doi.org/10.5114/aoms/120001

Copyright $\odot 2021$ Termedia \& Banach

\section{Abstract}

Introduction: Methylation of the $\mathrm{CpG}$ island in the promoter and its nearby area is one of the most important ways to inhibit gene expression. The aim of this study was to examine the potential relationship between CpG island methylation for fatty acid synthase (FASN) and sterol regulatory element-binding protein 1 (SREBP-1).

Material and methods: Ten newborn gerbil pups formed the NB group, 10 adult gerbils with a normal diet formed the control group, 10 adult gerbils with a high-fat diet formed the nonalcoholic fatty liver disease (NAFLD) group, and 10 8-month-old gerbils formed the aged group. Blood and liver samples were collected for serum lipid detection and histopathology. A pyrosequencing technique was employed to determine the methylation rate. Then, the transcription and expression levels for FASN and SREBP-1 were verified.

Results: Serum cholesterol and triglyceride were significantly increased in NAFLD and aged groups (vs. control, $p<0.05$ ). The gerbils in NAFLD and aged groups also showed obvious hepatic steatosis confirmed by histological examination. The control group had the highest methylation rates for FASN and SREBP-1, which were reduced in NAFLD and aged groups. Except for the NB group, both the transcription and expression levels of SREBP-1 and FASN genes were in the following order: control group > aged group $>$ NAFLD group. Genes of SREBP and FASN showed a trend of hypomethylation in the NAFLD gerbil model.

Conclusions: Expression of the SREBP gene tended to decrease, while expression of the FASN gene tended to increase with age and disease development. FASN and SREBP-1 methylation might be a new method to evaluate an NAFLD animal model and an available target for genetic marker screening.

Key words: nonalcoholic fatty liver disease, Mongolian gerbil, FASN, SREBP-1, CPG island methylation.

\section{Introduction}

Nonalcoholic fatty liver disease (NAFLD) is reported to be associated with epigenetic DNA methylation and nutritional status [1]. In adult mammals, $70 \%$ of DNA methylation happens in the CpG sites, while nearly $70-80 \%$ of CpGs genomes are methylated in humans [2]. DNA methylation mainly regulates gene expression through affecting the binding rates of genes and transcription factors [3]. One of the most important ways to inhibit gene expression is the dynamic methylation of CPG islands in the promoter as well as its nearby regions [4, 5]. More

\author{
Corresponding author: \\ Yuehuan Liu PhD \\ Hangzhou Medical College \\ 310013, Hangzhou, China \\ Phone: 0086-571-88215605 \\ E-mail:215193122@qq.com
}


than $1 / 3$ of genetic diseases caused by base conversion are attributed to cytidine methylation [6]. Mello et al. found 1,292 CpG methylations among 677 genes in the NASH population (non-alcoholic steatohepatitis, the second stage of NAFLD) [7]. Based on the analysis for 45,000 CpG loci, Ahrens et al. observed 467 dinucleotides with methylation in NASH patients in comparison to the non-obese population [8]. Additionally, they found that there were eight genes involved in NAFLD and metabolic disease whose CpG methylation was reversed [8].

In the NAFLD field, rat and mouse are the most common animals used in research, but they have many obvious disadvantages, such as a long modeling period (more than 48 weeks), a large difference in lipid metabolism compared to humans, and a low survival rate [9]. Our research group has been studying hyperlipidemia in a Mongolian gerbil model since 2005, and successfully established an NAFLD model in Mongolian gerbils [10]. NAFLD development in this animal has three distinctive stages (steatosis, steatohepatitis and fibrosis), mimicking the natural pathogenesis in humans. Moreover, the model could be available in a short time (1-12 weeks). Considering these outstanding improvements, the model received the Experimental Animal Science and Technology Achievement Award of China in 2016.

To study the underlying mechanism of NAFLD in Mongolian gerbils, two genes were selected in this research, fatty acid synthase (FASN) and sterol regulatory element-binding protein 1 (SREBP-1), which were crucial to lipid regulation and metabolism. We cloned part of the promoter sequence of both genes, detected the number and frequency of CpGs at specific locations by pyrosequencing and determined the expression by quantitative real-time PCR ( $\mathrm{PPCR}$ ) and western blot (WB) analysis, in order to investigate the potential mechanism from the perspective of epigenetic methylation.

\section{Material and methods}

\section{Animal models}

All the animals used in the study were male (Key Laboratory of Experimental Animal, Zhejiang Academy of Medical Sciences, China, Certificate No: SCXK (Zhejiang) 2014-0001, SYXK (Zhejiang) 2014-0008). Twenty 3-month-old gerbils (Meriones unguiculatus) of 50-70 g body weight were divided into a control group $(n=10)$ and an NAFLD model group $(n=10)$.

In addition, ten 8-month-old gerbils weighing 80-100 g were used as an aged group and ten newborn gerbils were used as an NB group. The NAFLD group was fed with a high-fat diet (7\% lard, $2 \%$ cholesterol, $0.5 \%$ bile salts and $10 \%$ yolk powder) while the other two groups were fed with a standard diet. Blood and liver were collected for further detection after 4-week feeding in control and NAFLD groups, while NB and aged groups were harvested at the same time. All the animals were sacrificed by anesthesia with carbon dioxide.

The use of animals in this study complied with all relevant and institutional policies and was approved by the Ethics Committee for Research on Laboratory Animal Use of Zhejiang Academy of Medical Sciences.

\section{Biochemical analysis and HE staining}

Serum (except the NB group) was extracted for lipid detection including total cholesterol (TC), triglycerides (TG), high-density lipoprotein (HDL) and low-density lipoprotein (LDL), determined by an automatic chemical analyzer (Autolab PM4000, Italy) with a commercial kit (Diasys Diagnostic Systems, Co. LTD., Shanghai, China) according to the manufacturer's instructions. One piece of liver tissue from the right lobe was used for the histological examination by HE staining.

\section{Capture of promoter regions in FASN and SREBP}

Primers were designed according to the sequences of human, rat and mouse as well as the amplification efficiency, fragment size and specificity. Primers were designed using Primer-blast software, and primer comparison was also performed using Blast in order to reduce the non-specific amplification. Gerbil liver (100 mg) was made into a TEN cell suspension, and then the genomic DNA was extracted with the dissolution in TE buffer solution ( $\mathrm{pH}$ 8.0) using the magnetic bead method. Next, the purity and concentration of extracted DNA were measured, with the 260/280 ratio > 1.6 indicating good quality. The reaction liquid was diluted into $100 \mathrm{ng} / \mu \mathrm{l}$ with TE buffer solution for the next assay at $4^{\circ} \mathrm{C}$. Primers for capturing the promoter regions in FASN and SREBP are listed in Table I. PCR Mix $(15 \mu l)$ contained $12.5 \mu \mathrm{l}$ KAPA2G Robust Mix, $1 \mu$ leach of forward and reverse primers $(10 \mathrm{mM}), 1 \mu$ l of template DNA and $9.5 \mu \mathrm{l}$ of $\mathrm{ddH}_{2} \mathrm{O}$. The thermal cycling profile for PCR was set up as follows: $94^{\circ} \mathrm{C}$ for $5 \mathrm{~min}$, $94^{\circ} \mathrm{C}$ for $30 \mathrm{~s}, \mathrm{Tm}$ for $30 \mathrm{~s}, 72^{\circ} \mathrm{C}$ for $1 \mathrm{~min}, 72^{\circ} \mathrm{C}$ for $5 \mathrm{~min}$, followed by heat preservation at $4^{\circ} \mathrm{C}$. PCR was performed on the Applied Biosystems 9700 PCR System.

\section{Detection of $\mathrm{CpG}$ islands in the promoter regions of FASN and SREBP}

DNA was treated with sulfite conversion for the methylation detection. To each sulfite mixture $800 \mu$ l of RNase-free $\mathrm{H}_{2} \mathrm{O}$ was added. PCR for sul- 
Table I. Primers for capturing the promoter regions in SREBP and FASN. F and R indicate the forward and reverse primer sequence

\begin{tabular}{|lccc|}
\hline Primer name & Primer sequence (5' to 3' $^{\prime}$ & TM & Length \\
\hline SREBP1-R1 & GGACACGCACCTTCGATGT & 62 & $1.8 \mathrm{~K}$ \\
\hline SREBP1-F1 & CCTGACCCTCCCTGGTTGAC & 64.2 & \\
\hline SREBP1-R2 & CCTGTGACTTAGGACAGGTAAAGC & 61.7 & $1.8 \mathrm{~K}$ \\
\hline SREBP1-F2 & GACTGCACAATGCTGAGCCT & 61.6 & \\
\hline FASN-R1 & TGAGGTTGGCCCAGAACTC & 61 & $1.0 \mathrm{~K}$ \\
\hline FASN-F1 & GGCCTCAGCGGAAGTCAT & 61 & \\
\hline FASN-R2 & TTGCCCAGGGCAGTTCAG & 63 & $1.0 \mathrm{~K}$ \\
\hline FASN-F2 & CGGCCTCGGTGTCCAATT & 64.4 & $1.0 \mathrm{~K}$ \\
\hline FASN-R3 & CCACATCGAWACCAATTGGAC & 62.4 & \\
\hline FASN-F3 & CAGTGTTCCCTATCCTGCCTACT & 61 & $1.2 \mathrm{~K}$ \\
\hline FASN-F4 & CCACGATGACCGGTAGTAAC & 58 & \\
\hline FASN-R4 & CGCTGGAGCACAAGGAAC & 60.6 & $1.2 \mathrm{~K}$ \\
\hline FASN-F5 & GGACAGAGATGAGGGCGTC & 60.8 & $1.5 \mathrm{~K}$ \\
\hline FASN-R5 & AGCCGTAGTCCAAGGAGAAG & 58.5 & \\
\hline FASN-F6 & GKCTGGGYGCCTCGTGGAT & 62.6 & \\
\hline FASN-R6 & AGCCGTAGTCCAAGGAGAAG & 58.5 &
\end{tabular}

fite conversion was performed in a total volume of $140 \mu \mathrm{l}$ containing $40 \mu \mathrm{l}$ of DNA solution (concentration range $1-500 \mathrm{ng} / \mu \mathrm{l}), 85 \mu \mathrm{l}$ of sulfite mixture and $15 \mu$ l of DNA protection solution. The PCR program was set up as follows: denaturation at $95^{\circ} \mathrm{C}$ for $5 \mathrm{~min}$, renaturation at $60^{\circ} \mathrm{C}$ for $25 \mathrm{~min}$, denaturation at $95^{\circ} \mathrm{C}$ for $5 \mathrm{~min}$, renaturation at $60^{\circ} \mathrm{C}$ for $85 \mathrm{~min}$, denaturation at $95^{\circ} \mathrm{C}$ for $5 \mathrm{~min}$, and renaturation at $60^{\circ} \mathrm{C}$ for $175 \mathrm{~min}$. Three cycles were performed between the intervals of renaturation. PCR products were kept at $20^{\circ} \mathrm{C}$ overnight. Next, DNA was purified with disulphonic acid solution using the adsorption column method, followed by elution in elution buffer.
The purified DNA was used directly in the next methylation-specific real-time PCR (MSP) analysis. MSP was performed in a total volume of $50 \mu$ l containing $10 \mu$ l of $5 \times$ buffer GC (KAPA), $1 \mu l$ of dNTP (10 mM/each), $1 \mu$ l each of upstream and downstream primers $(50 \mathrm{pM} / \mu \mathrm{l}), 2 \mu \mathrm{l}$ of template DNA and $0.2 \mu \mathrm{l}$ of Taq $(5 \mathrm{U} / \mu \mathrm{l})$. The thermal cycling profile for MSP was set up as follows: 40 cycles of $95^{\circ} \mathrm{C}$ for $3 \mathrm{~min}, 94^{\circ} \mathrm{C}$ for $30 \mathrm{~s}, 52^{\circ} \mathrm{C}$ for $30 \mathrm{~s}, 72^{\circ} \mathrm{C}$ for $1 \mathrm{~min}$, and a final $7 \mathrm{~min}$ at $72^{\circ} \mathrm{C}$. Primers for MSP of FASN1 and SREBP1 are listed in Table II.

Next, mixed beads together with PCR products formed a suspension, being immersed in $70 \%$ ethanol, 0.2 $\mathrm{M} \mathrm{NaOH}$ and flushing buffer to cool

Table II. Primer sequences for the methylation-specific PCR of FASN and SREBP. F and R indicate the forward and reverse primer sequence, and $S$ indicates the primer sequence of pyrosequencing

\begin{tabular}{|lcc|}
\hline Primer name & Primer sequence (5' to $\mathbf{3}^{\prime}$ ) & 5' modification \\
\hline 1.FASN-F6R1-1F & AGTAAGGATTTAGAAAGGTTTTAGTGG & \\
\hline 1.FASN-F6R1-1R & CTTAAAACCCCACCTCTTTACA & \\
\hline 1.FASN-F6R1-1S & AGAAAGGTTTTAGTGGAA \\
\hline 2.FASN-F6R1-2F & GGTTTTTAAGGTATTTGTTTAGGGTAGT & \\
\hline 2.FASN-F6R1-2R & CCAACCCCAAAAAACCAAATACTAATCT & \\
\hline 2.FASN-F6R1-2S & TTAGGGTAGTGTAGGT \\
\hline 3.SREBP1-1F & TTGGGAGGTGAGGAAAGTTATTT \\
\hline 3.SREBP1-1R & AAAACATTCTAACCCACAAATATCTAC \\
\hline 3.SREBP1-1S & GAGGTGAGGAAAGTTATTTA & \\
\hline 4.SREBP1-2F & 5GAGTAAAGAGTAGGTGGGTTTTTAT & \\
\hline 4.SREBP1-2R & AATTCAAACCCCTTATCTAAACCTAATTT \\
\hline 4.SREBP1-2S & GGTTTAGTGTTTGGGTA & \\
\hline
\end{tabular}


to room temperature. Next, the mixture was denatured at $85^{\circ} \mathrm{C}$ for $2 \mathrm{~min}$, and then the universal primer (cooled to room temperature) for sequencing (S primer) hybridized with the template DNA. Substrate mixture, enzyme mixture and 4 dNTP (QIAGEN) were added successively in the reagent chamber; the dosages were calculated according to the information of sequence design by PyroMark Assay Design software version 2.0. Finally, the reagent chamber together with the 96-hole reacting plate was put into the Pyrosequencing detector (PyroMark Q96 ID, QIAGEN) for the reaction. The methylation status of each site was automatically analyzed using Pyro Q-CpG software.

\section{FASN and SREBP-1 expression}

Quantitative real-time PCR ( $\mathrm{PPCR}$ ) was performed on the samples derived from liver tissue. For qPCR analysis, cDNA was synthesized from total RNA by reverse transcription with the RT-reagent Kit and DNA Eraser (TaKaRa Biotech, Kyoto, Japan). Primer synthesis of mRNA was designed using Primer 5.0 and Oli906.0, and $\beta$-actin was used as an internal control. qPCR reaction was performed in a total volume of $20 \mu$ l containing $10 \mu \mathrm{l}$ of $2 \times$ fluorescence qPCR Mix, $1 \mu \mathrm{l}$ each of upstream and downstream primers $(10 \mu \mathrm{M}), 1 \mu \mathrm{l}$ of template DNA and $7 \mu \mathrm{l}$ of $\mathrm{dd}_{2} \mathrm{O}$. The thermal cycling profile for $\mathrm{qPCR}$ was set up as follows: 40 cycles of pre-denaturation at $94^{\circ} \mathrm{C}$ for $1 \mathrm{~min}$, denaturation at $94^{\circ} \mathrm{C}$ for $10 \mathrm{~s}$, annealing at $59^{\circ} \mathrm{C}$ for $10 \mathrm{~s}$, followed by extension at $72^{\circ} \mathrm{C}$ for $10 \mathrm{~s}$. The results of $\mathrm{qPCR}$ were determined with the 2- $\Delta \Delta \mathrm{Ct}$ method. Primers for $\mathrm{qPCR}$ are listed in Table III.

For Western blot, protein lysates from liver were quantified by the BCA kit (Tiangen Biotech Co. Ltd., Beijing, China). $30 \mu \mathrm{g}$ of protein extracts was separated on $12 \%$ SDS-PAGE gels and transferred onto the PVDF membranes. After blocking with $5 \%$ non-fat milk, the membranes were incubated with primary antibody of FASN and SREBP-1 (1:500 dilution). Following secondary antibody in cubation and PBS washing, the bands were visu-

Table III. Primer sequences for qPCR of SREBP-1 and FASN. $F$ and $R$ indicate the forward and reverse primer sequence. $F$ and $R$ indicate the forward and reverse primer sequence

\begin{tabular}{|lcc|}
\hline Primer & \multicolumn{1}{c}{ Sequence $\left(5^{\prime}\right.$ to $\mathbf{3}^{\prime}$ ) } & Size \\
\cline { 1 - 2 }$\beta$-actin-F & CAGCCTTCCTTCCTGGGTAT & $105 \mathrm{bp}$ \\
\cline { 1 - 2 }$\beta$-actin-R & CTGTGTTGGCATAGAGGTCTT & \\
\cline { 1 - 2 } FASN-F & AGTGCTTCCCTTTCACGACT & $115 \mathrm{bp}$ \\
\hline FASN-R & GCCTGGGCTCAATAATAGTAGC & \\
\cline { 1 - 2 } SREBP-1-F & TTTCATTTCTCCGCTCCCAA & $120 \mathrm{bp}$ \\
\hline SREBP-1-R & TTCCTCAGCACGAAGCAAAG & \\
\hline
\end{tabular}

alized by X-ray exposure. Quantification was performed using the ImageJ software.

\section{Statistical analysis}

When applicable, results were presented as means \pm standard deviation (SD). Tamhane's T2 method was used for the comparison of serum lipid level and the paired $t$-test for gene expression. Correlation between the expression levels of FASN and SREBP and methylation rate were analyzed by Pearson's correlation test. All statistical analyses were performed using the SPSS software (version 13.0, SPSS Inc. Chicago, IL, USA). A two-sided $p<$ 0.05 was considered to be statistically significant.

\section{Results}

\section{Hepatic morphology}

In the gerbil NB group, the structure of the hepatic lobule was clear, the hepatic cord was neatly arranged, and the hematopoietic cells of extramedullary hematopoietic foci were observed in the hepatic sinusoid, in which large numbers of mononuclear cells and red cells were found (Figure 1 A). In the control group, no obvious fat deposition in the abdominal wall of the gerbils was observed; the liver was not obviously changed; it appeared red with sharp edges, clear lobules and plate-like arranged hepatic cords (Figure $1 \mathrm{~B}$ ). In the NAFLD and aged groups, the liver appeared yellow with blunt edges and a greasy feeling; some of the gerbils were observed to have a swollen spleen, in which the HE staining showed obvious hepatic steatosis, and the NAFLD group had more serious pathomorphology than the aged group, with hepatocyte necrosis and shedding widely distributed (Figures 1 C, D).

\section{Biochemical index}

The serum levels of TC, TG, HLD and LDL are given in Table IV. The serum TC level of the gerbil NAFLD group multiplied rapidly to 5.3 -fold higher compared to the gerbil control and aged groups, and the serum levels of HDL $(6.5$-fold) and LDL (4.3-fold) were also significantly increased $(p<$ 0.01 ). The serum TG level of the gerbil aged group was significantly higher than that of the gerbil control and NAFLD groups (5-fold, $p<0.01$ ). It indicates to some extent that the two types of fatty liver models are in different stages of NAFLD, or different types of hyperlipidemia.

\section{Sequences in the promoter regions of FASN and SREBP}

Two long fragments in the promoter regions of FASN and SREBP were captured. The sequences of the promoter regions of FASN and SREBP genes 

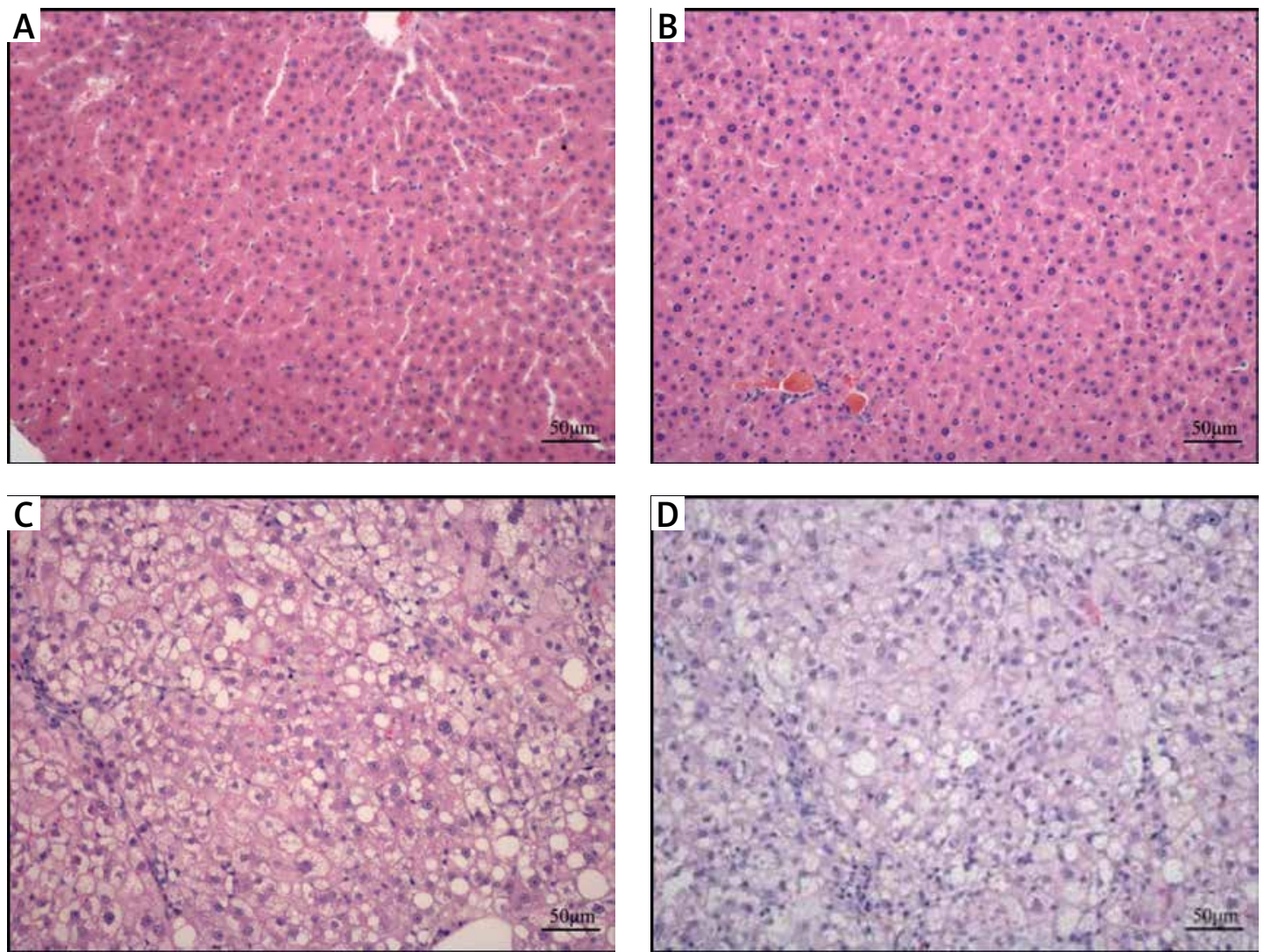

Figure 1. Pathology of liver in each group (HE staining $\times 20$ ). The histopathological modification in each treatment in NB group. The hepatic structure was neatly arranged, and the hematopoietic cells of extramedullary hematopoietic foci were observed in the hepatic sinusoid, in which a large number of mononuclear cells and red cells were found (A). In the control, no obvious fat deposition in the abdominal wall of the gerbils was observed; the liver was not obviously changed; it appeared red with sharp edges, clear lobules and plate-like arranged hepatic cords (B). In the NAFLD and aged groups, the liver appeared yellow with blunt edges and a greasy feeling; some of the gerbils had a swollen spleen, in which the HE staining showed obvious hepatic steatosis (C, D)

Table IV. Serum levels of biochemical index for each group (mmol/L)

\begin{tabular}{|lcccc|}
\hline Group & TC & HDL & LDL & TG \\
\hline NB & - & - & - & - \\
\hline Control & $2.55 \pm 0.70^{\mathrm{B}}$ & $0.81 \pm 0.28^{\mathrm{B}}$ & $1.26 \pm 0.83^{\mathrm{B}}$ & $0.83 \pm 2.02^{\mathrm{B}}$ \\
\hline NAFLD & $17.30 \pm 2.51^{\mathrm{A}}$ & $7.47 \pm 0.34^{\mathrm{A}}$ & $8.30 \pm 1.59^{\mathrm{A}}$ & $1.59 \pm 0.36^{\mathrm{B}}$ \\
\hline Aged & $3.23 \pm 1.09^{\mathrm{B}}$ & $1.14 \pm 0.28^{\mathrm{B}}$ & $1.90 \pm 0.55^{\mathrm{B}}$ & $8.37 \pm 3.48^{\mathrm{A}}$ \\
\hline
\end{tabular}

A,Bindicates significant difference $(p<0.01)$.

referred to the attached sequence data (Supplementary Table SI).

\section{Number of $\mathrm{CpG}$ island in the gerbil liver}

The methylation levels showed dramatic differences in different regions of the same promoter region of genes. For the FASN gene, the methylation rate was highest in the gerbil control group, followed by the gerbil NAFLD and aged group $(p<0.05)$. For the SREBP gene, the methylation rate was also highest in the gerbil control group, yet followed by the gerbil aged and NAFLD group $(p<0.05)$. The results above indicated that methylation levels of FASN and SREBP gradually decreased in NAFLD with the supplementation of a high-fat diet (except the gerbil NB group), which was closely associated with hyperlipidemia and NAFLD (Figures 2, 3).

\section{Transcription and expression levels of FASN and SREBP}

In the transcription level, the FASN gene was highest in the gerbil NB group, followed by the gerbil aged, NAFLD and control groups. Yet, for the SREBP gene, the transcription level was highest in the gerbil control group, followed by the gerbil NB, aged and NAFLD groups (Figure 4). The expression levels of FASN and SREBP genes are presented in 
Well: A4

Assay: FASN-F6R1-1S

Sample ID: 4

Sequence to analyze: ATTATTYGGATTYGGTTGTAATATTGGATGAGTYGAGTGTYGGGTTTTTTT Analysis parameters have been edited.

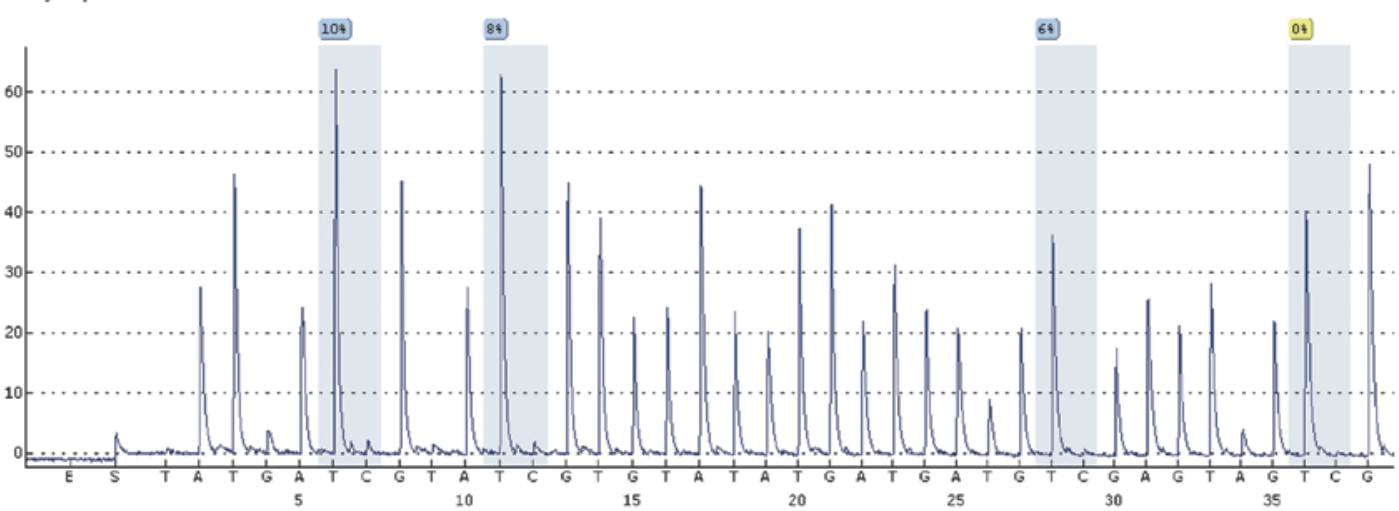

Well: A5

Assay: FASN-F6RI-IS

Assay: FASN 5

Sequence to analyze: ATTATTYGGATTYGGTTGTAATATTGGATGAGTYGAGTGTYGGGTTTTTTI Analysis parameters have been edited.

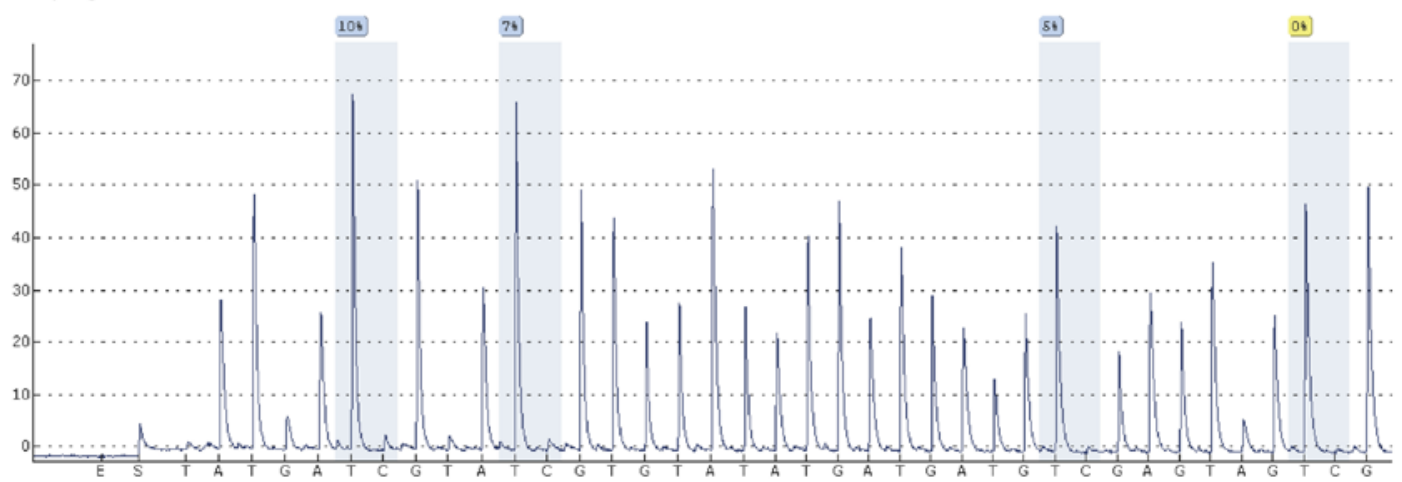

Figure 2. Detection results of CPG island in the promoter region of FASN and SREBP. The CpG sites were measured using pyrosequencing, and the "methylation\%" was the average of "\%" of each CpG site measured. The figure presents the results of the methylation rate of samples $A 4, A 5, E 1, E 2$

Figure 5. For the FASN gene, the expression level was highest in the gerbil aged group, followed by the gerbil NAFLD, NB and control groups. Yet, for the SREBP gene, the expression level was highest in the gerbil control group, followed by the gerbil aged, NB and NAFLD groups.

The correlation between gene expression and methylation rate was computed. For the FASN gene, the methylation rate was negatively correlated with the expression level in the gerbil NB $(r=-0.78)$ and control groups $(r=-0.61)$, while this trend weakened in the gerbil NAFLD $(r=$ $-0.11)$ and aged groups $(r=0.04)$, which was consistent with the results of WB analysis. For the SREBP gene, positive and negative correlations between methylation rate and expression level were found in the gerbil NB $(r=0.22)$ and NAFLD groups $(r=-0.29)$, respectively. However, based on high methylation rates, the expression levels in the gerbil aged $(r=0.05)$ and control groups $(r=-0.07)$ tended to be weakened with the increase of methylation rates.

\section{Discussion}

The methyl of DNA methylation is mainly derived from the methionine, folic acid and choline in food. High-fat diet feeding (methyl-donor-deficient diet, choline and L-amino acids) for animals can affect the gene expression through the change of DNA methylation. Methylenetetrahydrofolate reductase (MTHFR) is a key enzyme in the metabolic process of folic acid, which is involved in the methionine metabolic cycle and DNA methylation through converting the 5,10-methyltetrahydrofolate (MTHF) into 5-MTHF [11]. Folic acid deficiency can interfere with the metabolism of methionine, resulting in a decreased methyl donor in the body; therefore, hypomethylation occurs in the genome of the liver, promoting liver injury [12]. The above is the main reason for inducing fat deposition in the liver of animals [13], and also an important theory for establishing the high-fat diet-induced animal model. In the study conducted by Wang et al., progress in pathological changes of NAFLD (fatty liverfibrosis-NASH) were found to be closely associated 
Well: EI

ample ID: 1

SREBP1

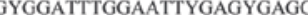

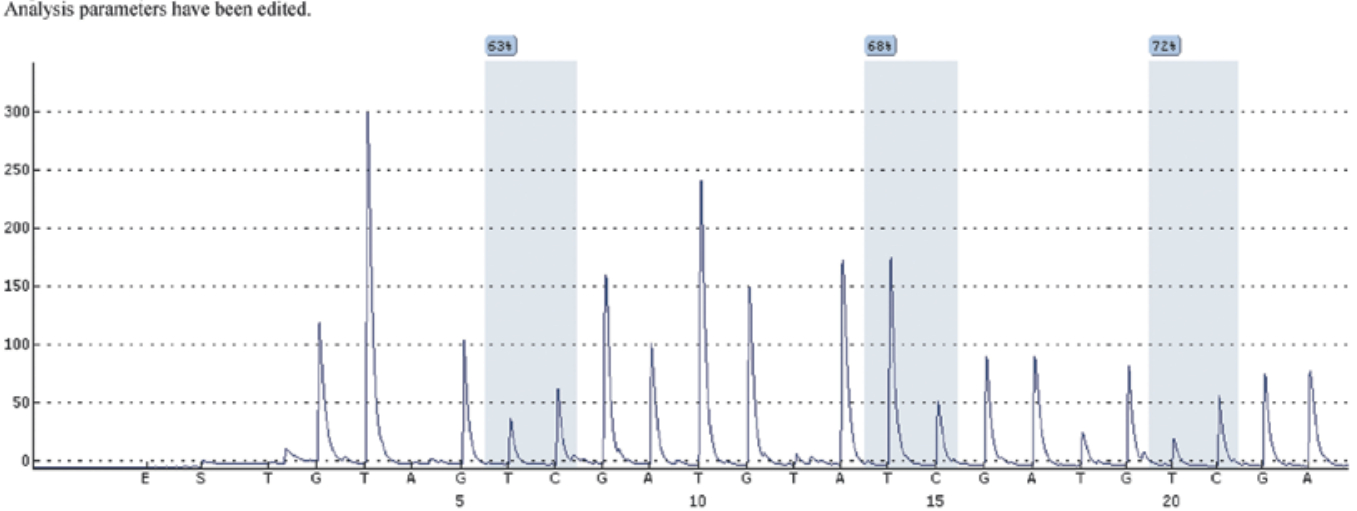

Well: E2

Assay: SREBPI-1S

Sample ID: 2

Sequence to analyze: GTTTGYGGATTTGGAATTYGAGYGAGG

Analysis parameters have been edited.

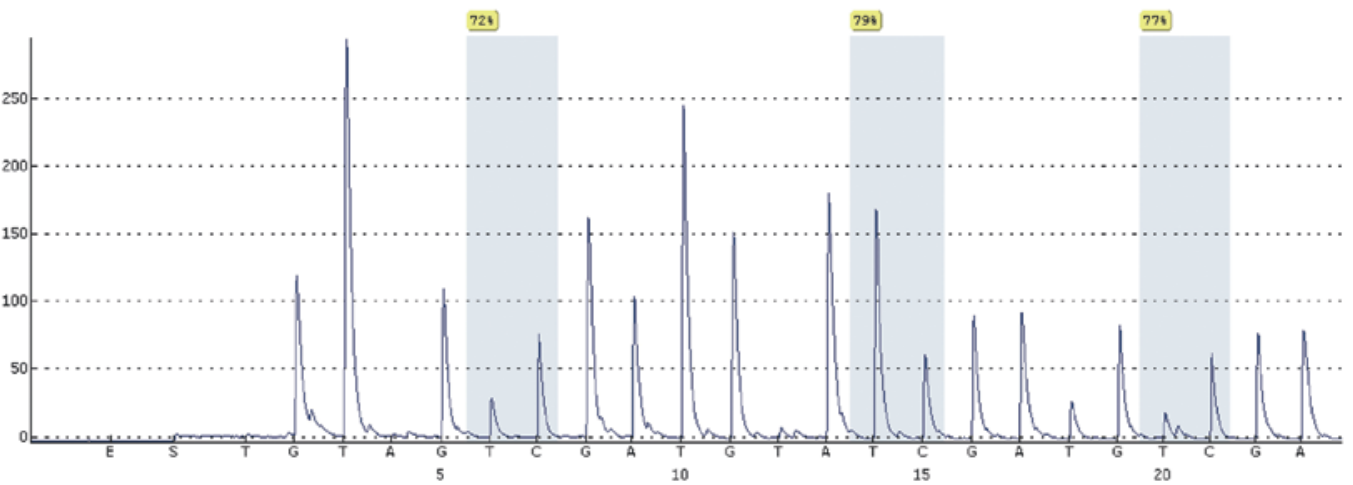

Figure 2. Cont.
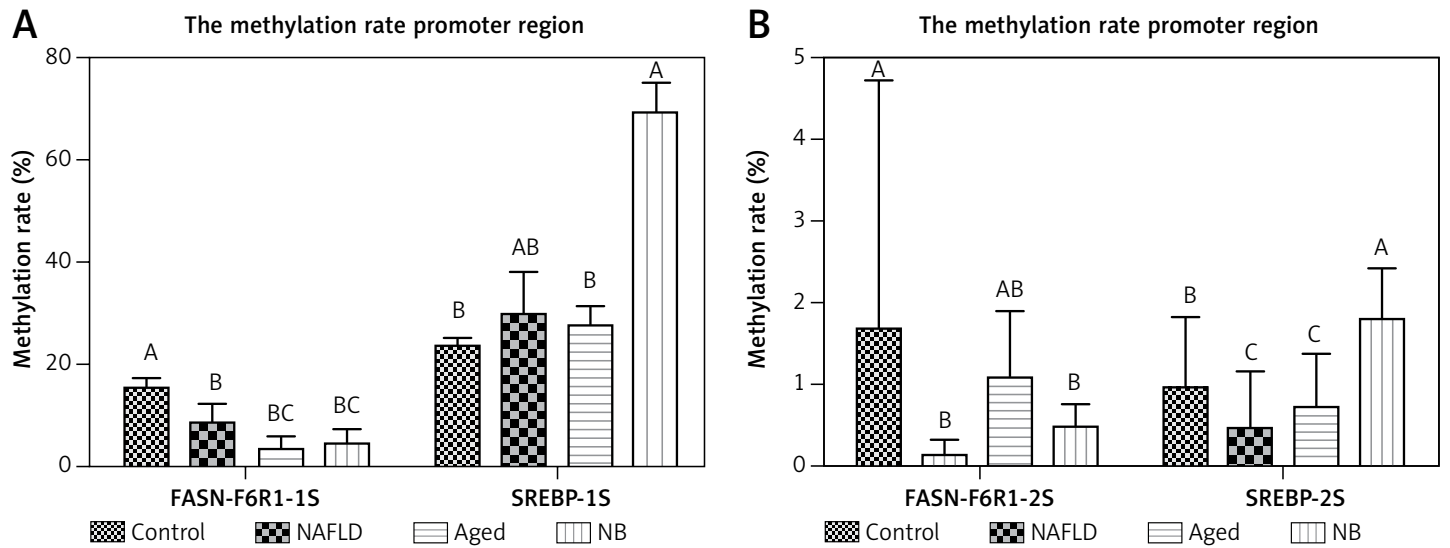

Figure 3. Methylation rate in the promoter regions of FASN and SREBP. A - The high methylation rate region of FASN and SREBP genes of each treatment. B - The low methylation rate region of the FASN gene and SREBP gene of each treatment. The letters $A / B / C$ above each column indicate a significant difference $(p<0.01)$

with the alteration of genome methylation in the liver, which was related to the gradually decreased methyl donors due to the disorder of the methionine cycle [14]. Metabolic syndrome (including hyperlipidemia) is often associated with a balance disorder between methyl supplement and methyl consumption [15]. In addition, the change of DNA methylation not only accumulates as time proceeds, but also is influenced by eating habits, body weight, aging and the environment [16, 17]. Epigenomic studies of mouse models indicated that a high-fat diet and exercise can change the lipid 
A

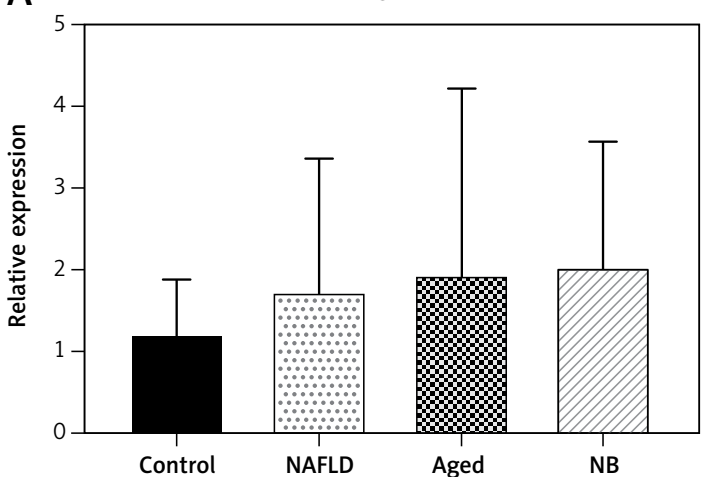

B

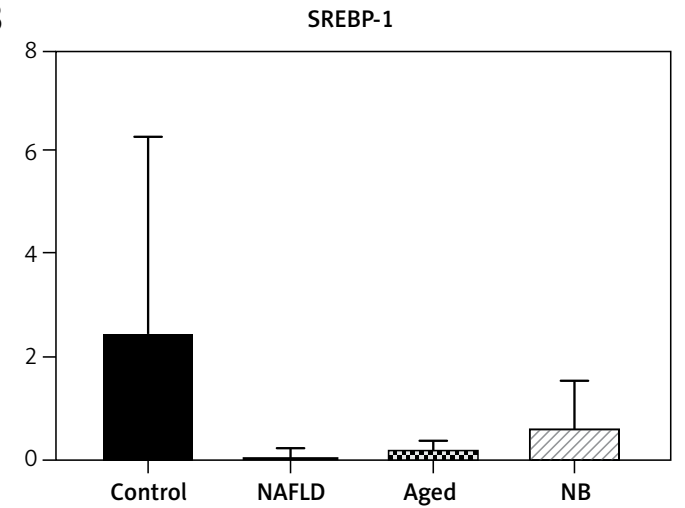

Figure 4. Transcription levels of SREBP and FASN (qPCR). A - The transcriptional level of the FASN gene for newborn, aged, NAFLD, and normal. B - The transcriptional level of the SREBP gene for newborn, aged, NAFLD, and normal gerbils

A

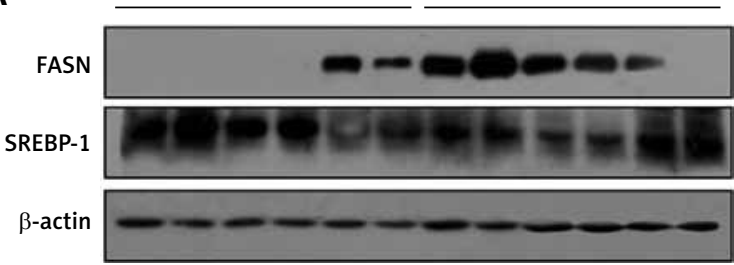

B

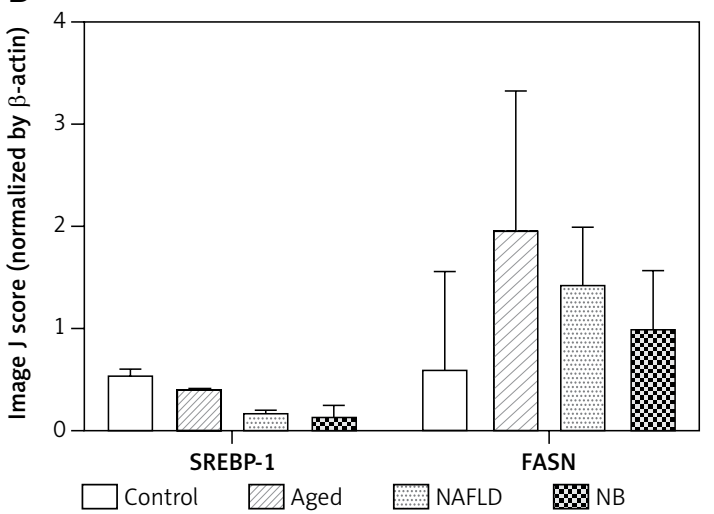

metabolism pathway and process of muscle development, of which exercise can significantly change the gene hypermethylation caused by a high-fat diet [18]. Fujiki et al. found that the DNA methylation of a specific gene (PPAR $\gamma$ ) could change the expression of specific genes in adipocytes [19], while Kovalenko et al. found that drug-induced (pyrazinamide) liver injury was related to the decrease of overall DNA methylation level in the liver genome, with a dose-dependent effect [20]. Therefore, it could be speculated that the fatty liver of gerbils with NAFLD (induced model) was not only related to the changes of DNA methylation caused by the high-fat diet, but also related to the time cumulative effect of DNA methylation and aging (8-month-old gerbil model).

The methylation levels showed dramatic differences in the different regions of the same promoter region of the gene. For the FASN gene,
NB

NAFLD

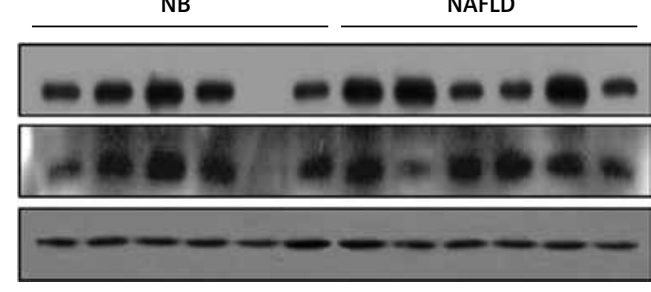

Figure 5. Expression levels of SREBP and FASN (Western blot analysis). A - The Western blot results of FASN and SREBP genes for each treatment. $\mathbf{B}$ - The average gray value of each treatment compared with $\beta$-actin

the number of CPG islands selected from the two segments FASN-6R1-1S and FASN-6R1-2S showed a different distribution in each group. The dynamic methylation rate changes of these groups were affected by diet, age and NAFLD progression. Hypermethylation in the gerbil control group was sig nificantly different than in the gerbil NAFLD and aged groups $(p<0.05)$; for the SREBP gene, the methylation rate was also higher in the gerbil control group compared to the gerbil aged and NAFLD groups $(p<0.05)$. The results above indicated that methylation levels of FASN and SREBP genes gradually decreased in NAFLD with the addition of a high-fat diet except the newborn gerbils (the overall DNA methylation levels in the liver genome are relatively high, inhibiting the expressions of lipogenic genes, which may be closely related to the reprogramming and environmental factors at the embryonic stage) [21, 22]. 
Sterol regulatory element-binding protein 1 plays an important role in the metabolism of lipid and protein in the liver cells; it is a key molecule regulating the lipid metabolism and expressions of genes related to the synthesis of fatty acid and TG [23]. In this study, the transcription level of the SREBP gene was higher in the gerbil control group compared to the gerbil aged and NAFLD groups (except the gerbil NB group), which was highly consistent with the gene expression levels. The results above indicated that the gene expression levels tended to decrease with the formation of NAFLD, which seemed to be inconsistent with the function of lipogenic genes. However, a study conducted in geese showed that a high-fat diet could reduce the expression level of SREBP mRNA [24, 25], with a significant negative correlation between the expression levels of the SREBP gene in liver tissues and liver weight index $(p<0.01)[25,26]$, which may be due to the excessive deposition of TG in the liver resulting in an adaptive feedback of the goose body, thus inhibiting the abundance of SREBP and reducing the expressions of genes related to lipid synthesis [26].

Fatty acid synthase is a key enzyme in fatty acid synthesis, which has binding regions SRE (sterol regulatory elements) of SREBP-1C and ChREBP (carbohydrate responsive-element binding protein) in the promoter region. So the dynamic changes of SREBP can regulate the expression of FASN. Also, insulin can induce the tri-dimensional hypermethylation of $\mathrm{H} 3 \mathrm{~K} 4$ histone and super acetylation of $\mathrm{H} 3$ and $\mathrm{H} 4$ of FASN to regulate expression of the FASN gene, and can also accelerate expression of the FASN gene by enhancing the combination of SREBP-1C and ChREBP [27]. In this study, the transcription and expression levels of the FASN gene were both higher in the gerbil aged group compared to the gerbil NAFLD and control groups, indicating that the expression levels tended to increase with the formation of NAFLD, which was consistent with the pathogenesis of NAFLD including the increase in synthesis of fatty acid and cholesterol, abnormal transport of TC and cholesterol, and inhibition of fatty acid oxidation decomposition in the liver [28].

In view of the susceptibility of the Mongolian gerbil to a high-fat diet and differences in blood lipids between the age groups as well as the combination of results in the present study, we concluded that the liver genomic DNA of gerbils was influenced by a high-fat diet and age, which may be different in methylation level in multiple gene expression or signal transduction pathways. The present study provided a basis for future research on NAFLD in gerbils.

\section{Acknowledgments}

This research was supported by Zhejiang Provincial Natural Science Foundation of China (LY16H030011), Science and Technology Department of Zhejiang Province (2011C37096, 2015C37102), and the Health and Family Planning Commission of Zhejiang Province (2015RCA006, 2018KY345).

\section{Conflict of interest}

The authors declare no conflict of interest.

\section{References}

1. Lee JH, Friso S, Choi SW. Epigenetic mechanisms underlying the link between non-alcoholic fatty liver diseases and nutrition. Nutrients 2014; 6: 3303-25.

2. Boers R, Boers J, de Hoon B, et al. Genome-wide DNA methylation profiling using the methylation-dependent restriction enzyme LpnPI. Genome Res 2018; 28: 88-99.

3. Fila M, Pawłowska E, Blasiak J. Mitochondria in migraine pathophysiology - does epigenetics play a role? Arch Med Sci 2019; 15: 944-56.

4. Rozenberg JM, Shlyakhtenko A, Glass K, et al. All and only $\mathrm{CpG}$ containing sequences are enriched in promoters abundantly bound by RNA polymerase II in multiple tissues. Bmc Genomics 2008; 9: 67.

5. Christopher MA, Kyle SM, Katz DJ. Neuroepigenetic mechanisms in disease. Epigenetics Chromatin 2017; 10: 47.

6. Zheng Y, Joyce BT, Liu L, et al. Prediction of genome-wide DNA methylation in repetitive elements. Nucleic Acids Res 2017; 45: 8697-711.

7. de Mello VD, Matte A, Perfilyev A, et al. Human liver epigenetic alterations in non-alcoholic steatohepatitis are related to insulin action. Epigenetics 2017; 12: 287-95.

8. Ahrens M, Ammerpohl O, von Schonfels W, et al. DNA methylation analysis in nonalcoholic fatty liver disease suggests distinct disease-specific and remodeling signatures after bariatric surgery. Cell Metab 2013; 18: 296-302.

9. Temmerman AM, Vonk RJ, Niezen-Koning K, Berger R, Fernandes J. Effects of dietary cholesterol in the Mongolian gerbil and the rat: a comparative study. Lab Anim 1989; 23: 30-5.

10. Liu YH, Yu CH, Li YM. A new model: genetic characterizations of nonalcoholic fatty liver disease in Mongolian gerbil. Hepatol Int 2017; 11 (Suppl. 1): S1-S1093.

11. Shimizu K, Onishi M, Sugata E, et al. Disturbance of DNA methylation patterns in the early phase of hepatocarcinogenesis induced by a choline-deficient L-amino acid-defined diet in rats. Cancer Sci 2007; 98: 1318-22.

12. Halsted CH, Villanueva JA, Devlin AM, et al. Folate deficiency disturbs hepatic methionine metabolism and promotes liver injury in the ethanol-fed micropig. Proc Natl Acad Sci USA 2002; 99: 10072-7.

13. Houten SM, Argmann CA. New driver for lipid synthesis. Cell 2011; 147: 719-21.

14. Wang LJ, Zhang HW, Zhou JY, et al. Betaine attenuates hepatic steatosis by reducing methylation of the MTTP promoter and elevating genomic methylation in mice fed a high-fat diet. J Nutr Biochem 2014; 25: 329-36.

15. Li D, Tian YJ, Guo J, et al. Nicotinamide supplementation induces detrimental metabolic and epigenetic changes in developing rats. Br J Nutr 2013; 110: 2156-64. 
16. Jiang MH, Fei J, Lan MS, et al. Hypermethylation of hepatic Gck promoter in ageing rats contributes to diabetogenic potential. Diabetologia 2008; 51: 1525-33.

17. Cordero P, Campion J, Milagro FI, Martinez JA. Dietary supplementation with methyl donor groups could prevent nonalcoholic fatty liver. Hepatology 2011; 53: 2151-2.

18. Zhou D, Hlady RA, Schafer MJ, et al. High fat diet and exercise lead to a disrupted and pathogenic DNA methylome in mouse liver. Epigenetics 2017; 12: 55-69.

19. Fujiki K, Kano F, Shiota K, Murata M. Expression of the peroxisome proliferator activated receptor gamma gene is repressed by DNA methylation in visceral adipose tissue of mouse models of diabetes. Bmc Biol 2009; 7: 38

20. Kovalenko VM, Bagnyukova TV, Sergienko OV, et al. Epigenetic changes in the rat livers induced by pyrazinamide treatment. Toxicol Appl Pharmacol 2007; 225 293-9.

21. Reik W, Dean W, Walter J. Epigenetic reprogramming in mammalian development. Science 2001; 293: 1089-93.

22. Ehara T, Kamei Y, Takahashi M, et al. Role of DNA methylation in the regulation of lipogenic glycerol-3-phosphate acyltransferase 1 gene expression in the mouse neonatal liver. Diabetes 2012; 61: 2442-50.

23. Shimomura I, Shimano H, Horton JD, Goldstein JL, Brown MS. Differential expression of exons $1 \mathrm{a}$ and $1 \mathrm{c}$ in mRNAs for sterol regulatory element binding protein-1 in human and mouse organs and cultured cells. J Clin Invest 1997; 99: 838-45.

24. Han CC, Wang JW, Pan ZX, et al. Effect of cholesterol on lipogenesis and VLDL-TG assembly and secretion in goose primary hepatocytes. Mol Cell Biochem 2013; 374: 163-72.

25. Liu X, Li P, He C, Qu X, Guo S. Comparison of overfed Xupu and Landes geese in performance, fatty acid composition, enzymes and gene expression related to lipid metabolism. Asian-Australas J Anim Sci 2020; 33: 1957-64.

26. Walker AK, Jacobs RL, Watts JL, et al. A conserved SREBP-1/phosphatidylcholine feedback circuit regulates lipogenesis in metazoans. Cell 2011; 147: 840-52.

27. Du X, Cai C, Yao J, Zhou Y, Yu H, Shen W. Histone modifications in FASN modulated by sterol regulatory element-binding protein $1 \mathrm{c}$ and carbohydrate responsive-element binding protein under insulin stimulation are related to NAFLD. Biochem Biophys Res Commun 2017; 483: 409-17.

28. Shimpi PC, More VR, Paranjpe M, et al. Hepatic lipid accumulation and Nrf2 expression following perinatal and peripubertal exposure to bisphenol $\mathrm{A}$ in a mouse model of nonalcoholic liver disease. Environ Health Perspect 2017; 125: 87005 . 